\title{
ASYMPTOTIC SOLUTION FOR SIMPLE BIPED WALKER MODEL
}

\author{
S.A. Yudaev, D.I. Rachinskii \\ Samara National Research University, Samara, Russia \\ University of Texas at Dallas
}

\begin{abstract}
In this paper, we study a simple passive dynamic biped robot with point feet and legs without knee. The mathematical model describing the robot is a switched system, which includes an inverted double pendulum. Robot's gait and its stability depend on parameters such as the slope of the ramp, mass distribution for the robot, and the length of its legs. The main result of the paper is an asymptotic solution for the gait.
\end{abstract}

Keywords: biped robot, passive dynamic walking.

Keywords: Citation: Yudaev SA, Rachinskii DI. Asymptotic solution for simple biped walker model. CEUR Workshop Proceedings, 2016; 1638: 709-716. DOI: 10.18287/1613-0073-2016-1638-709-716

\section{Introduction}

Passive Dynamic Walkers (PDW) have been widely developed since 1990 when they were introduced [1]. The problem of legged robot locomotion continues to generate interest of researchers attempting to improve the design of walkers.

Most of us do walking as a daily routine without any thought or care. Nevertheless, walking is a complex process depending on various factors. Human body, controlled by nervous system, involves skeletal muscles and limbs to reproduce an efficient and natural gait. From there, biped gait dynamics are examined by several disciplines. Passive walkers constitute a class of robots that use gravity to power their dynamics. Several experimental studies have shown that this kind of walking is possible with reasonable stability over a range of slopes without any actuation [2], [3].

In this paper, a simple biped model is considered. This model is an extension of the model that has been comprehensively analyzed in [4]. We include point masses of the legs and allow the positions of these masses to be changed. Analytic results are complemented by, and compared to, numerical simulations. 


\section{Model description}

Figure 1 represents a simple passive walker we in two dimensions. Given appropriate initial conditions and placed at the top of the ramp with a small slope, this robot starts moving.

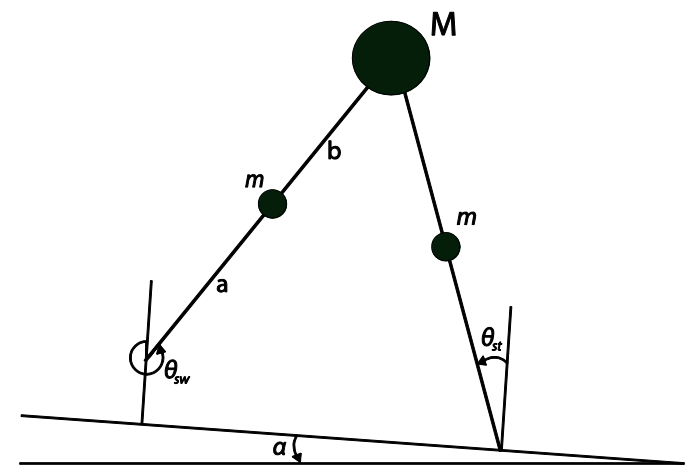

Fig. 1. Sketch of a simple biped robot on a slope

Angles $\theta_{s w}, \theta_{s t}$ represent the values of angles for swing and stance leg respectively. Ground slope denoted as $\alpha$.We consider kneeless legs of mass $m$ and length $l$ connected through a hip joint of mass $M$. We do not include any friction force at the leg joint. Variables $a, b$ are responsible for the leg masses distribution. As far as we consider point feet, extra mass on the toe is not included.

Another assumption of model is that ground is perfectly rigid, and foot collision is an absolutely plastic collision. As a consequence of this, the moment of impact and foot transition is instant. At the time of impact, the vertical distance between foot of swing leg and the walking surface will become a zero, so it met the constraint $\left(\theta_{s t}-\alpha\right)+\left(\theta_{s w}-\alpha\right)=0$. The stance foot is assumed fixed on the ground until impact occurs, then foots rename and the stance leg become a swing leg and vice versa. Period of motion between foot transition is a gait cycle.

\section{Equations of motion}

The equations of motions are well-known. We based our research on the work of passive walking robot by Nita H. Shah, Mahesh A. Yeolekar [5]. We only made some substitutions to reduce the number of parameters. First is $\beta=m / M$, and another one is $\mu=a / l, v=b / l$. This way, one gait cycle is derived in a second order system:

$$
M(\theta) \ddot{\theta}+N(\theta, \dot{\theta}) \dot{\theta}+G(\theta, \alpha)=0,
$$


where $\theta=\left(\theta_{s t}, \theta_{s w}\right)^{t}$ is the vector of angular coordinates; $M(\theta)$ represents the inertia matrix; the matrix $N(\theta, \dot{\theta})$ accounts for centrifugal and Coriolis forces; and, $G(\theta, \alpha)$ contains the gravity terms:

$$
\begin{aligned}
& M(\theta)=\left[\begin{array}{cc}
\beta \mu^{2}+1+\beta & -\beta v \cos \left(\theta_{s t}-\theta_{s w}\right) \\
-\beta v \cos \left(\theta_{s t}-\theta_{s w}\right) & \beta v^{2}
\end{array}\right], \\
& N(\theta, \dot{\theta})=\left[\begin{array}{cc}
0 & -\beta v \sin \left(\theta_{s t}-\theta_{s w}\right) \dot{\theta}_{s w} \\
\beta v \sin \left(\theta_{s t}-\theta_{s w}\right) \dot{\theta}_{s t} & 0
\end{array}\right], \\
& G(\theta, \alpha)=\left[\begin{array}{c}
\frac{g}{l}(-\beta \mu-1-\beta) \sin \left(\theta_{s t}-\alpha\right) \\
\frac{g}{l} \beta v \sin \left(\theta_{s w}-\alpha\right)
\end{array}\right] .
\end{aligned}
$$

Since centrifugal and Coriolis forces affects the walker motion only slightly, we will ignore the $N(\theta, \dot{\theta})$ matrix. Then, equation (1) becomes:

$$
M(\theta) \ddot{\theta}+G(\theta, \alpha)=0
$$

or, componentwise:

$$
\begin{aligned}
& \left(\beta \mu^{2}+1+\beta\right) \ddot{\theta}_{s t}(t)-\beta v \cos \left(\theta_{s t}(t)-\theta_{s w}(t)\right) \ddot{\theta}_{s w}(t)+\frac{g}{l}(-\beta \mu-1-\beta) \sin \left(\theta_{s t}(t)-\alpha\right)=0, \\
& -\cos \left(\theta_{s t}(t)-\theta_{s w}(t)\right) \ddot{\theta}_{s t}(t)+v \ddot{\theta}_{s w}(t)+\frac{g \sin \left(\theta_{s w}(t)-\alpha\right)}{l}=0 .
\end{aligned}
$$

Next, to describe the switch of stance and swing legs, we use the algebraic transition equation $\theta^{+}=J \theta^{-}$with

$$
J=\left[\begin{array}{ll}
0 & 1 \\
1 & 0
\end{array}\right]
$$

which relates the pre-impact and post-impact coordinate values. Here, the "- " and " +" indices denote the state variables before and after the impact, respectively. In addition, the conservation of the angular momentum gives to the following relation between the pre- and post-impact angular velocities:

$\dot{\theta}^{+}=K(\varphi) \dot{\theta}^{-}$,

where

$$
\theta_{s w}-\theta_{s t}=\varphi, K(\varphi)=\left[V^{+}(\varphi)\right]^{-1} V^{-}(\varphi),
$$




$$
\begin{aligned}
& V^{-}(\varphi)=\left[\begin{array}{cc}
-\beta \mu \nu+(2 \beta \mu+1) \cos \varphi & -\beta \mu \nu \\
-\beta \mu \nu & 0
\end{array}\right], \\
& V^{+}(\varphi)=\left[\begin{array}{cc}
\beta \mu^{2}+1+\beta-\beta v \cos (\varphi) & \beta v^{2}-\beta v \cos \varphi \\
-\beta v \cos \varphi & \beta v^{2}
\end{array}\right] .
\end{aligned}
$$

We reproduced some numerical results from [5] for the hybrid model. Figure 2 presents a gait cycle of equation (2):

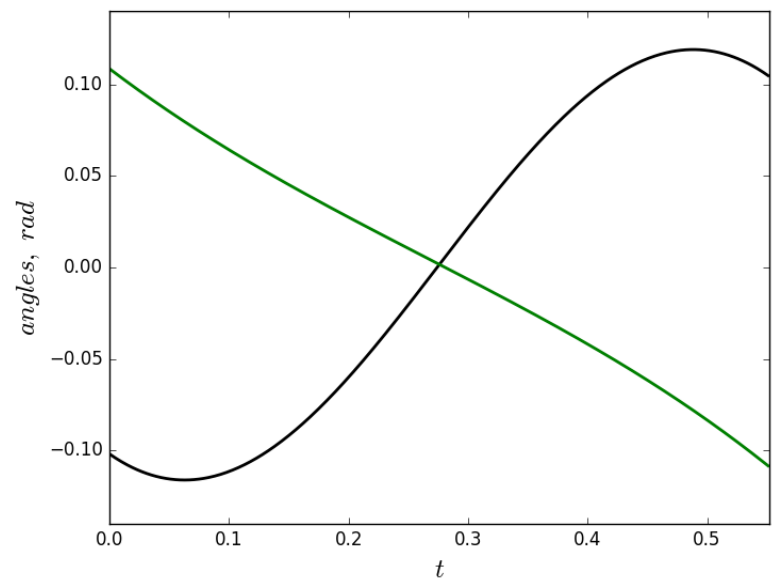

Fig. 2. Leg angles at a typical gait cycle, $\beta=0.1, \mu=0.7, \nu=0.3, \alpha=0.35$

Figure 3 shows phase space diagrams. The parameters are the same as in Figure 2.

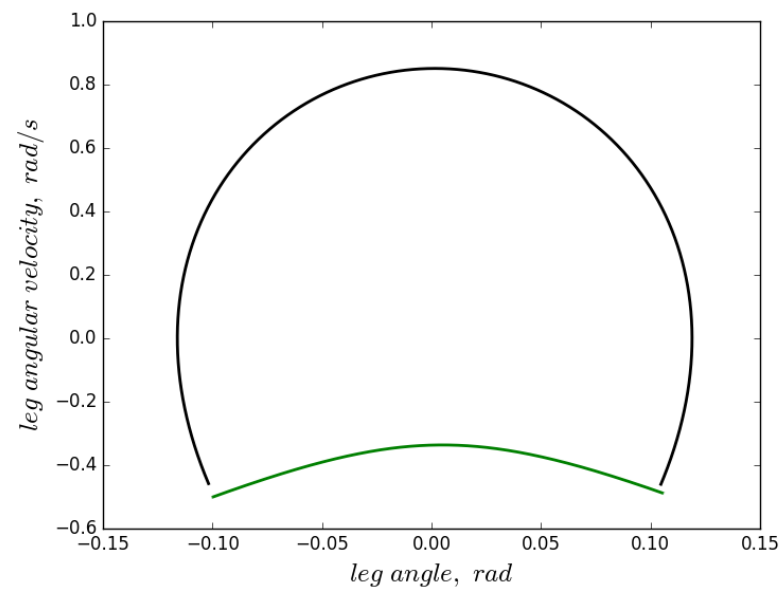

Fig. 3. Phase space diagram 


\section{Asymptotic Solution}

We use perturbation method in order to study walking cycles as $\alpha \rightarrow 0$. Following [4], we define the scaling parameter $\varepsilon$ and scaled variables $\theta_{s t}, \theta_{s w}$ by:

$$
\begin{aligned}
& \alpha=\varepsilon^{3}, \\
& \theta(t)=\varepsilon \Theta_{s t}(t), \\
& \dot{\theta}_{s t}(t)=\varepsilon \dot{\Theta}_{s t}(t), \\
& \theta_{s w}(t)=\varepsilon \Theta_{s w}(t), \\
& \dot{\theta}_{s t}(t)=\varepsilon \dot{\Theta}_{s t}(t) .
\end{aligned}
$$

Substituting these equations in (2) and expanding in a power series with respect to the small parameter $\varepsilon$ gives us two governing equations with no zero order terms. After dividing both equations by $\varepsilon$, the expansion contains only even powers of $\varepsilon$. Therefore, we can make a substitution $\varepsilon^{2}=\delta$, which results in:

$$
\begin{aligned}
& \left(\beta \mu^{2}+1+\beta\right) \ddot{\Theta}_{s t}(t)-\beta v \ddot{\Theta}_{s w}(t)+\frac{g}{l}(-\beta \mu-1-\beta) \Theta_{s t}(t)+ \\
& +\left(\frac{1}{2} \beta v\left(\Theta_{s t}(t)-\Theta_{s w}(t)\right)^{2} \ddot{\Theta}_{s w}(t)-\frac{g}{l}\left(-\beta \mu-\frac{1}{l}-\frac{\beta}{l}\right)\left(1+\frac{1}{6} \Theta_{s t}(t)^{3}\right)\right) \delta=0, \\
& -\ddot{\Theta}_{s t}(t)+v \ddot{\Theta}_{s w}(t)+\frac{g}{l} \Theta_{s w}(t)+ \\
& +\left(\frac{1}{2}\left(\Theta_{s t}(t)-\Theta_{s w}(t)\right)^{2} \ddot{\Theta}_{s t}(t)-\frac{g}{l}\left(1+\frac{1}{6} \Theta_{s w}(t)^{3}\right)\right) \delta=0 .
\end{aligned}
$$

We assume power series solutions of the form:

$$
\Theta_{s t}(t)=\Theta_{s t 0}(t)+\delta \Theta_{s t 1}(t)+\ldots, \quad \Theta_{s w}(t)=\Theta_{s w 0}(t)+\delta \Theta_{s w 1}(t)+\ldots,
$$

and initial conditions of the form:

$$
\begin{aligned}
& \Theta_{s t}(0)=\Theta_{s t 0}(0)+\delta \Theta_{s t 1}(0)+\ldots, \quad \dot{\Theta}_{s t}(0)=\dot{\Theta}_{s t 0}(0)+\delta \dot{\Theta}_{s t 1}(0)+\ldots \\
& \Theta_{s w}(0)=\Theta_{s w 0}(0)+\delta \Theta_{s w 1}(0)+\ldots, \quad \dot{\Theta}_{s w}(0)=\dot{\Theta}_{s w 0}(0)+\delta \dot{\Theta}_{s w 1}(0)+\ldots .
\end{aligned}
$$

Using (6),(7) together with (4),(5), we obtain zero-order equations and the first correction equations. For $\beta=0, \delta=0$, from (5), (6) we obtain the zero-order equations: 


$$
\begin{aligned}
& \ddot{\Theta}_{s t 0}(t)-\frac{g}{l} \Theta_{s t 0}(t)=0, \\
& -\ddot{\Theta}_{s t 0}(t)+v \ddot{\Theta}_{s w 0}(t)+\frac{g}{l} \Theta_{s w 0}(t)=0 .
\end{aligned}
$$

Solutions to equations (8),(9) are:

$$
\begin{aligned}
& \Theta_{s t 0}(t)=\frac{1}{2 \sqrt{g}}\left(\sqrt{g} \Theta_{s t 0}(0)+\sqrt{l} \dot{\Theta}_{s t 0}(0)\right) e^{\frac{\sqrt{g} t}{\sqrt{l}}}+\frac{1}{2 \sqrt{g}}\left(\sqrt{g} \Theta_{s t 0}(0)-\sqrt{l} \Theta_{s t 0}(0)\right) e^{-\frac{\sqrt{g} t}{\sqrt{l}}}, \\
& \Theta_{s w 0}(t)=\frac{1}{\sqrt{g}(v+1)} \sin \left(\frac{\sqrt{g} t}{\sqrt{l} \sqrt{v}}\right) \sqrt{l} \sqrt{v}\left(\dot{\Theta}_{s w 0}(0) v+\dot{\Theta}_{s w 0}(0)-\dot{\Theta}_{s t 0}(0)\right)+ \\
& +\frac{1}{v+1} \cos \left(\frac{\sqrt{g} t}{\sqrt{l} \sqrt{v}}\right)\left(\Theta_{s w 0}(0) v+\Theta_{s w 0}(0)-\Theta_{s t 0}(0)\right)+ \\
& +\frac{1}{\sqrt{g}(2 v+2)}\left(\left(\sqrt{g} \Theta_{s t 0}(0)+\sqrt{l} \dot{\Theta}_{s t 0}(0)\right) e^{\frac{2 \sqrt{g} t}{\sqrt{l}}}-\dot{\Theta}_{s t 0}(0) \sqrt{l}+\sqrt{g} \Theta_{s t 0}(0)\right) e^{\frac{-\sqrt{g} t}{\sqrt{l}}} .
\end{aligned}
$$

Equations for the first correction are obtained by substituting (6) into (4), (5) with $\beta=0$ :

$\ddot{\Theta}_{s t 1}(t)-\frac{g}{l} \Theta_{s t 1}(t)+\frac{g}{l}+\frac{1}{6} \frac{g}{l} \Theta_{s t 0}(t)^{3}=0$,

$\frac{g}{l} \Theta_{s w 1}(t)-\frac{g}{l}-\Theta_{s t 0}(t) \Theta_{s w 0}(t) \ddot{\Theta}_{s t 0}-\frac{1}{6} \frac{g}{l} \Theta_{s w 0}(t)^{3}+v \ddot{\Theta}_{s w 1}(t)+$

$+\frac{1}{2} \Theta_{s t 0}(t)^{2} \ddot{\Theta}_{s t 0}(t)+\frac{1}{2} \Theta_{s w 0}(t)^{2} \ddot{\Theta}_{s t 0}(t)-\ddot{\Theta}_{s t 1}(t)=0$.

Equations (10), (11) are nonlinear. Using the zero order approximation $\Theta_{s t 0}(t), \Theta_{s w 0}(t)$, we could solve for the corrections $\Theta_{s t 1}(t), \Theta_{s w 1}(t)$.

Similarly, we get a beta-assumption equation for stance leg:

$$
\begin{aligned}
& \mu^{2} \ddot{\Theta}_{s t 0}(t)+\ddot{\Theta}_{s t 1}(t)+\ddot{\Theta}_{s t 0}(t)-v \ddot{\Theta}_{s w 0}(t)-\frac{g \mu}{l} \Theta_{s t 0}(t)- \\
& -\frac{g}{l} \Theta_{s t 1}(t)-\frac{g}{l} \Theta_{s t 0}(t)=0 .
\end{aligned}
$$


Now, we can plot the solution of equations (8)-(12) in the form:

$\Theta_{s t}(t)=\Theta_{s t 0}(t)+\varepsilon \Theta_{s t 1}(t)+\beta \Theta_{s t 1}$,

$\Theta_{s w}(t)=\Theta_{s w 0}(t)+\varepsilon \Theta_{s w 1}(t)$,

and compare with numerical results. Figure 4 shows the comparison.

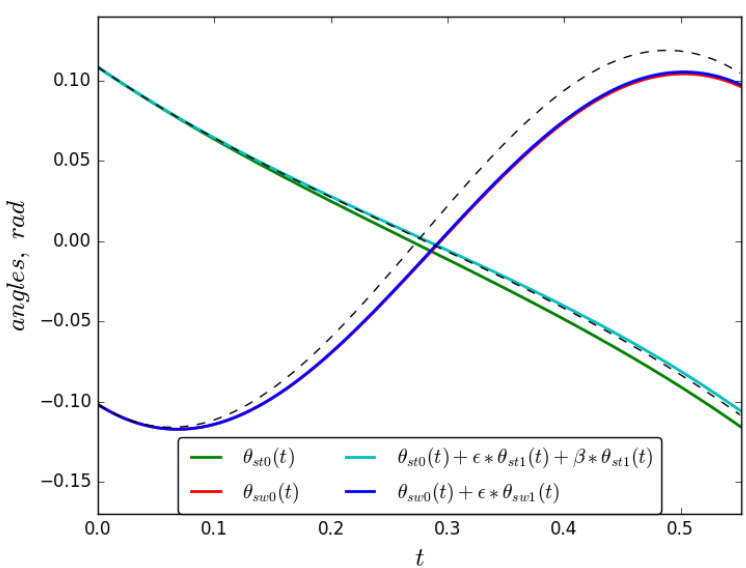

Fig. 4. Leg angles over gait cycle. Dotted lines represent numerical result. Colored lines are for asymptotic

\section{Conclusion}

We have carried out a research about numerical and asymptotic solution for simple walker. We have concluded in the paper that analytic solution can precise the numerical result even with the only first correction.

\section{Acknowledgements}

This work is supported in part by the Russian Foundation for Basic Research (grant 14-01-97018-p) and the Ministry of Education and Science of the Russian Federation under the Competitiveness Enhancement Program of Samara University (2013-2020).

\section{References}

1. McGeer T. Passive dynamic walking. International Journal of Robotics Research, 1990; 9(2): 62-82. 
2. Spong MW, Bhatia G. Further results on control of the compass gait biped. International Conference on Intelligent Robots and Systems, 2003: 1933-1938.

3. Goswami A, Espiau B, Keramane A. Limit cycles and their stability in a passive bipedal gait. IEEE Conference on Robotics and Automation, 1996: 246-251.

4. Garcia M, Chatterjee A, Ruina A, Coleman MJ. The Simplest Walking Model: Stability, Complexity, and Scaling. ASME Journal of Biomechanical Engineering, 1998; 120(2): 281-228.

5. Shah N, Yeolekar M. Influence of Slope Angle on the Walking of Passive Dynamic Biped Robot. Applied Mathematics, 2015; 6 (3): 456-465. 